\title{
Búsqueda de sensaciones y hábitos de tabaquismo, consumo de alcohol y práctica deportiva en estudiantes de Educación Secundaria
}

\author{
Pedro Ángel Latorre Román, ' José Carlos Cámara Pérez, ${ }^{1}$ Felipe García Pinillos ${ }^{1}$
}

Artículo original

\section{SUMMARY}

Objective

The aim of this study is to analyze the relationship between sensation seeking activity, consumption of alcohol and/or tobacco, and sport practice in teenagers.

\section{Methodology}

In this study, there were 272 participants, all currently attending high school (age $=14.14 \pm 1.35$ years): 130 boys and 142 girls; 93 first year students and 179 second year students. The Sensation Seeking Scale (EBS-J) of Pérez et al. (1987) was used as well as an adaptation of the Health Behavior in School-Aged Children (Currie, 1998), which analyzed lifestyle.

\section{Results}

$76 \%$ of the boys and $75.4 \%$ of the girls are reported to have consumed alcoholic beverages $(p=0.906) ; 36.2 \%$ of the boys and $42.3 \%$ of the girls are reported to have consumed tobacco $(p=0.304)$; and $92.3 \%$ of the boys and $57.7 \%$ of the girls $(p<0.001)$ are reported to have been athletes, a practice which is reduced with age $(p=0.009)$. Age is a risk factor for tobacco and alcohol consumption; however, sex is not indicative of this consumption. Sensation seeking leads to the consumption of alcohol and tobacco because it creates a necessity for excitement and inhibition. No dimension of the EBS-J scale predicted a sedentary lifestyle.

\section{Conclusions}

BS is a personality factor related to the early consumption of substances such as alcohol and tobacco and whose traits were revealed as good indicators of the propensity in teenagers to consume these substances.

Key words: Teenagers, sports, alcohol, snuff consumption, sensation seeking.

\section{RESUMEN}

\section{Objetivo}

Analizar la relación existente entre la búsqueda de sensaciones y el consumo de alcohol, tabaco y práctica deportiva en adolescentes.

\section{Metodología}

Los participantes de este estudio han sido 272 escolares de educación secundaria (edad= $14.14 \pm 1.35$ años), 130 niños y 142 niñas, 93 alumnos del primer ciclo y 179 del segundo ciclo. Se empleó la Escala de Búsqueda de Sensaciones (EBS-J) de Pérez et al. (1987) y para el análisis de los hábitos de vida se utilizó una adaptación del Health Behaviour in School-Aged Children (Currie, 1998).

\section{Resultados}

El $76 \%$ de los niños y el $75.4 \%$ de las niñas se declaran bebedores de alcohol $(p=0.906)$; el $36.2 \%$ de los niños y el $42.3 \%$ de las niñas fuman $(p=0.304)$; $y$ el $92.3 \%$ de los niños por el $57.7 \%$ de las niñas $(p<0.001)$ son deportistas, práctica que se reduce con la edad $(p=0.009)$. La edad es un factor de riesgo de tabaquismo y consumo de alcohol; por el contrario, el sexo no es un factor predictivo de estos consumos. La búsqueda de excitación y desinhibición son factores moderadamente predictivos del consumo de tabaco y la desinhibición igualmente en el consumo de alcohol. Ninguna dimensión de la EBS-J mostró capacidad predictiva de la conducta sedentaria.

\section{Conclusiones}

La BS es un factor de personalidad relacionado con el consumo temprano de sustancias como el alcohol y el tabaco, revelándose algunos de sus rasgos como factores predictivos del consumo de estas sustancias en adolescentes.

Palabras clave: Adolescentes, práctica deportiva, consumo de alcohol, consumo de tabaco, búsqueda de sensaciones.

Departamento de Didáctica de la Expresión Musical, Plástica y Corporal. Universidad de Jaén, España.

Correspondencia: Pedro Ángel Latorre Román. Baja de San Jorge 15, Úbeda (Jaén). España, 23400, Móvil: 606308265, E-mail: platorre@ujaen.es

Recibido primera versión: 5 de septiembre de 2013. Segunda versión: 6 de noviembre de 2013. Aceptado: 13 de enero de 2014. 


\section{INTRODUCCIÓN}

La adolescencia es una etapa de cambios muy importantes en el ámbito afectivo, social, mental y físico, que va configurando la personalidad del adolescente, sus intereses, conductas y estilos de vida. En la adolescencia, los individuos suelen tener más independencia y se incrementan los tiempos sin supervisión adulta continua, lo que puede influir en la realización y adquisición de conductas de riesgo. La adolescencia es la etapa decisiva en la adquisición de estilos de vida, ya que se consolidan algunas tendencias comportamentales adquiridas en la infancia y se incorporan otras nuevas provenientes de otros entornos sociales de influencia. ${ }^{1}$

Los factores que habitualmente se han investigado en población adolescente sobre estilos de vida saludables hacen referencia a la práctica de actividad físico-deportiva, el consumo de tabaco y de alcohol. ${ }^{2-5}$

Actualmente en España existe una prevalencia importante de obesidad y sobrepeso en niños y jóvenes. La prevalencia de obesidad entre los niños que no practican ningún deporte habitualmente es más elevada en comparación con los que sí tienen este hábito. ${ }^{6}$ En el estudio AVENA ${ }^{7}$ se destaca, a su vez, que un $40.8 \%$ de los adolescentes españoles indican que no practican actividad física, y que los varones son más activos que las mujeres $(\mathrm{p}<0.001)$.

El consumo de tabaco y alcohol es muy común entre los adolescentes y se ha convertido en un problema de salud pública. En España, según el Observatorio Español sobre Drogas, ${ }^{8}$ en 2008, en estudiantes de educación secundaria de entre 14 y 18 años, un 81.2\% había tomado bebidas alcohólicas alguna vez en la vida; un $44.6 \%$, tabaco; un $35.2 \%$, cannabis y un $17.3 \%$, tranquilizantes o pastillas para dormir. Con respecto al consumo de tabaco, se ha reducido especialmente en las adolescentes: en 2008 el porcentaje de chicas que fumaban diariamente bajó hasta el 16.4\% frente al $24 \%$ que lo hacía en 2004. Sin embargo, con el alcohol la situación es diferente, pues ha aumentado el consumo de riesgo y el porcentaje de adolescentes que reporta beber hasta emborracharse es del 29\%. La edad de inicio al consumo, tanto de tabaco como de alcohol, se ha mantenido estable entre 2004 y 2008: en 13.7 años en el inicio del alcohol y 13.3 años en el de tabaco. ${ }^{8}$ A su vez, en el estudio AVENA $^{7}$ se indica que un $29.9 \%$ de los adolescentes señala consumir tabaco habitualmente, no existiendo diferencias en función del sexo. Una revisión realizada por Cándido et al. (2007) ${ }^{9}$ a partir de cinco estudios nacionales y 52 internacionales desarrollados en la última década, relativos a la identificación de los factores de riesgo y protección asociados al consumo de tabaco y alcohol en estudiantes de educación secundaria, destaca que el uso y abuso de drogas legales como el alcohol y el tabaco se encuentran relacionados con el consumo recíproco de estas sustancias y el consumo de drogas ilegales, variables personales (etnia, género, edad, curso académico, rasgos de personalidad, tipo de creencias, actitudes y expectativas frente al consumo y trastornos internalizantes versus externalizantes), el entorno social y familiar (estatus socioeconómico, rendimiento académico, consumo de drogas legales e ilegales por familiares e iguales, estilos parentales, calidad en las relaciones familiares y simultaneidad de estudios y trabajo) y la organización de los hábitos recreacionales del fin de semana.

La práctica de actividad física se ha considerado como una alternativa para prevenir hábitos de vida insanos. Pero esta creencia generalizada de que la participación en actividades deportivas limita el uso de sustancias adictivas como el alcohol no está demasiado clara y muchos deportistas jóvenes presentan patrones de consumo de dicha sustancia similar o significativamente mayor que sus pares sedentarios. En algunos casos, la práctica habitual de actividad física es un elemento que se asocia a una menor prevalencia en el consumo de alcohol. ${ }^{10}$ En otros casos, la participación deportiva se relaciona con el consumo de alcohol más alto. ${ }^{11}$ Sin embargo y en relación con el tabaco, Tercedor et al. $(2007)^{7}$ destacan que tanto en varones como en mujeres, los adolescentes activos manifiestan un menor consumo de tabaco ( $\mathrm{p} \leq 0.01)$ que los sedentarios y que, cuanto mayor edad, mayor consumo de tabaco y menor práctica de actividad física, tanto en varones como en mujeres $(p<0.001)$. Existe por tanto una tendencia a fumar en menor cantidad o incluso a no hacerlo por parte de los sujetos físicamente activos. Un $80.9 \%$ de los sujetos activos señalan no fumar frente al $71.4 \%$ de los no activos. En un estudio realizado con adolescentes entre 15 y 18 años de la Comunidad Valenciana, Pastor et al. $(2006)^{12}$ concluyeron que la competencia deportiva ejerce una influencia indirecta sobre las conductas de salud, y que la participación deportiva actúa como variable mediadora en esta relación. Así, en ambos sexos, cuanto mayor es la percepción de competencia deportiva, mayor es la práctica de deporte y menor es el consumo de tabaco y de alcohol.

La búsqueda de sensaciones (BS) ha sido estudiada ampliamente en la investigación sobre la propensión a tomar riesgos en una amplia gama de comportamientos como la conducción de riesgo, comportamientos que contribuyen a las lesiones no intencionales, el consumo de alcohol, de tabaco y de drogas ilícitas, los malos hábitos alimenticios, la inactividad física, la práctica de deportes de riesgo, las conductas sexuales, la delincuencia u otras conductas antisociales y otros hábitos recreacionales. ${ }^{13-16}$ Zuckerman (1979) ${ }^{17}$ considera que la búsqueda de sensaciones supone una necesidad de experimentar variadas y complejas sensaciones y el deseo de correr riesgos físicos y sociales, por el simple deseo de disfrutar de tales experiencias. Estos rasgos se pueden dividir en cuatro dimensiones: búsqueda de emoción y de aventura, búsqueda de experiencias, desinhibición y susceptibilidad al aburrimiento. ${ }^{18}$ Los hombres experimentan mayor BS que las mujeres, lo que puede deberse tanto a factores biológicos como a factores de socialización. En cuanto a la edad parece existir una relación negativa entre edad 
y BS. ${ }^{15,19}$ La BS se asocia con diversas variables biológicas, incluyendo neurotransmisores, enzimas y hormonas ${ }^{15}$ y la variación individual puede ser heredable. ${ }^{20}$

La BS se correlaciona por tanto con el consumo de alcohol y tabaco entre los jóvenes. ${ }^{21,22}$ En adolescentes, los consumidores de sustancias adictivas como el tabaco, el alcohol o la marihuana puntúan más alto en BS que los no consumidores. ${ }^{23}$ A su vez, la búsqueda de emociones y sensaciones se ha destacado en deportistas practicantes de deportes de riesgo. ${ }^{24}$ Oliva (2007) ${ }^{25}$ señala que la corteza prefrontal, estructura fundamental en muchos procesos cognitivos, experimenta un importante desarrollo a partir de la pubertad que no culmina sino hasta los primeros años de la adultez temprana. Otros cambios afectan al circuito mesolímbico, relacionado con la motivación y la búsqueda de recompensas, que va a verse influido por las alteraciones hormonales asociadas a la pubertad. Como consecuencia, durante la adolescencia se produce un cierto desequilibrio entre ambos circuitos cerebrales que puede justificar el aumento de la impulsividad y las conductas de riesgo durante esta etapa.

La identificación temprana de conductas de riesgo de los adolescentes, como el consumo de tabaco y alcohol, y el sedentarismo, es esencial para reducir las consecuencias negativas para la salud. El objetivo de este estudio es analizar la relación existente entre la búsqueda de sensaciones y el consumo de alcohol y tabaco, y la práctica deportiva en adolescentes.

\section{MÉTODO \\ Participantes}

Los participantes de este estudio son escolares de educación secundaria, estudiantes que cursan de $1^{\circ}$ a $4^{\circ}$ de Educación Secundaria Obligatoria (ESO) de la provincia de Jaén (España). El total de sujetos ha sido de 272 estudiantes (edad=14.14 \pm 1.35 años), 130 niños (edad=14.11 \pm 1.55 años, Índice de Masa Corporal [IMC] $=21.19 \pm 3.70 \mathrm{~kg} / \mathrm{m}^{2}$ ) y $142 \mathrm{ni}-$ ñas (edad=14.13 \pm 1.61 años, IMC=21.32 $\pm 3.47 \mathrm{~kg} / \mathrm{m}^{2}$ ), de 1 os cuales 93 estudiantes son del primer ciclo de ESO y 179 del segundo ciclo.

\section{Materiales}

Se ha empleado la Escala de Búsqueda de Sensaciones para Niños y Adolescentes (EBS-J) de Pérez et al. (1987). ${ }^{26}$ Esta escala mide el rasgo de BS en niños y adolescentes; consta de 50 reactivos en una escala de respuesta dicotómica, y se divide en cuatro dimensiones: búsqueda de emociones (BEM), búsqueda de excitación (BEX), desinhibición (DES), susceptibilidad al aburrimiento (SAB), sinceridad (S) y una valoración global de la escala (Total escala EBS-J). Para el análisis de los hábitos de vida se ha empleado una adapta- ción del Health Behaviour in School-Aged Children ${ }^{27}$ en el que se recogen aspectos sobre consumo de alcohol (sí/no) y su frecuencia (nunca, rara vez, todos los días, todas las semanas, todos los meses), así como la frecuencia de borracheras (nunca, una vez, 2 a 3 veces, 4 a 10 veces, más de 10 veces). El consumo de tabaco (sí/no) y su frecuencia (nunca, todos los días, varias veces a la semana pero no todos los días, menos de una vez a la semana), la práctica deportiva (sí/no) y su frecuencia (todos los días, 4-6 veces a la semana, 2-3 veces a la semana, una vez a la semana, una vez al mes, menos de una vez al mes, nunca); estableciéndose el punto de corte para considerar al adolescente como deportista el que practica deporte como mínimo tres veces a la semana, teniendo en cuenta los umbrales de práctica deportiva saludable señalados por Haskell et al. (2007). ${ }^{28}$ Además, se registraron las horas de práctica deportiva a la semana. Como parámetros antropométricos se analizaron: la altura $(\mathrm{cm})$ que se midió con un estadiómetro Seca 22 (Hamburgo, Alemania), la masa corporal (kg) que se registró con una báscula Seca 634 (Hamburgo, Alemania) y el IMC que se obtuvo de la ecuación $\mathrm{IMC}=\operatorname{peso}(\mathrm{kg}) /$ talla $\left(\mathrm{m}^{2}\right)$.

\section{Procedimiento}

Se mantuvo una entrevista con el director del centro escolar para exponer los objetivos de la investigación y promover su colaboración. Posteriormente se envió una carta informativa a los padres para explicar los objetivos del estudio y solicitar su consentimiento informado por escrito donde autorizaban a sus hijos a participar en el estudio. Las encuestas fueron cumplimentadas por los alumnos en sus aulas, de forma voluntaria, anónima y con la presencia de un investigador para atender sus dudas. La toma de datos se realizó entre abril y mayo de 2012. El estudio fue aprobado por el Comité de Bioética de la Universidad de Jaén.

\section{Análisis estadístico}

Los datos fueron analizados mediante el programa estadístico SPSS, v.19.0 para Windows (SPSS Inc., Chicago, USA) y el nivel de significación se fijó en $p<0.05$. Los datos se muestran en estadísticos descriptivos de media, desviación típica y porcentajes. Se establecieron como factores explicativos el ciclo educativo, el sexo, bebedor vs. no bebedor, fumador vs. no fumador y deportista vs. no deportista. La prueba chi cuadrada se empleó para analizar las variables cualitativas entre grupos. Se realizaron ANOVAs entre grupos, ANCOVA con el sexo como covariable al comparar grupos de edad y ANCOVA con edad como covariable al comparar sexos. Se realizó regresión logística binaria con la escala EBS-J, la edad y el sexo como predictores de los hábitos de tabaquismo, consumo de alcohol y conducta sedentaria. La eficacia predictiva de las variables anteriores en cada hábito de vida se ha establecido mediante curvas ROC (del inglés: Receiver Operating 
Cuadro 1. Consumo de bebidas alcohólicas y de tabaco en deportistas y no deportistas

\begin{tabular}{lccccc}
\hline & $\begin{array}{c}\text { Deportistas } \\
\mathrm{n}(\%)\end{array}$ & $\begin{array}{c}\text { No deportistas } \\
\mathrm{n}(\%)\end{array}$ & p-valor \\
\hline Bebedor de alcohol & & & & & \\
- No & 56 & $(27.7)$ & 11 & $(15.7)$ & 0.044 \\
- Sí & 146 & $(72.3)$ & 59 & $(84.3)$ & \\
Fumador & & & & & \\
- No & 132 & $(65.3)$ & 33 & $(47.1)$ & 0.007 \\
- Sí & 70 & $(34.7)$ & 37 & $(52.9)$ & \\
\hline
\end{tabular}

Characteristic curves). Se realizó análisis cluster k-medias con la escala EBS-J. Por último, se realizaron correlaciones Pearson y Spearman entre la escala EBS-J y la frecuencia de consumo de tabaco, alcohol y horas de práctica deportiva.

\section{RESULTADOS}

En relación con la práctica deportiva existen diferencias significativas $(\mathrm{p}<0.001)$ entre sexos, destacándose que el 92.3\% de los niños por el $57.7 \%$ de las niñas son deportistas. En el consumo de alcohol, el 76\% de los niños y el 75.4\% de las niñas se declaran consumidores de alcohol ( $p=0.906)$. En el consumo de tabaco, el $36.2 \%$ de los niños y el $42.3 \%$ de las niñas fuman ( $p=0.304)$. Por ciclos educativos, el $83.9 \%$ de los niños del primer ciclo de la ESO por el $69.3 \%$ del segundo ci- clo se declaran deportistas ( $\mathrm{p}=0.009)$. En el consumo de alcohol, el $45.7 \%$ del primer ciclo de la ESO por el $91.1 \%$ del segundo ciclo se declaran consumidores de alcohol ( $\mathrm{p}<0.001)$. Por último, en relación con el hábito de fumar, el 15.1\% del primer ciclo de la ESO por el 52\% del segundo ciclo declaran ser fumadores $(\mathrm{p}<0.001)$. En la frecuencia de consumo de alcohol, el 3.2\% por el 14.5\% ( $<<0.001)$ de los niños de primer ciclo y segundo ciclo de la ESO respectivamente, consumen semanalmente bebidas alcohólicas. En relación con el sexo, no hay diferencias significativas ( $\mathrm{p}=0.413$ ); así, el $11.5 \%$ de los niños y el 9.9\% de las niñas declaran beber alcohol todas las semanas. En los fumadores y en relación con el sexo no hay diferencias significativas en el número de cigarrillos semanales consumidos $(\mathrm{p}=0.429)$; los niños consumen $6.88 \pm 22.76$ cigarros por $9.26 \pm 26.51$ cigarros las niñas. Por ciclos educativos sí encontramos diferencias significativas ( $\mathrm{p}<0.001)$ : los niños de primer ciclo consumen 1.72 \pm 10.97 por $11.45 \pm 28.98$ cigarros semanales en segundo ciclo. En la frecuencia de borracheras, no encontramos diferencias significativas ( $p=0.780)$ entre sexos: el $65.4 \%$ de los niños por el $59.9 \%$ de las niñas nunca se ha emborrachado. Por ciclos educativos sí existen diferencias significativas $(\mathrm{p}<0.001)$ : el $86 \%$ por el $50.3 \%$ de los niños de primer y segundo ciclo de la ESO, respectivamente, no se ha emborrachado nunca.

En el cuadro 1 se muestran las contingencias que relacionan el hábito de la práctica deportiva con el hábito del tabaquismo y consumo de bebidas alcohólicas. Se puede observar que el porcentaje de bebedores de alcohol y fumadores es mayor en el grupo de no deportistas.

Cuadro 2. Escala EBS-J en relación con los diferentes factores explicativos (ciclo educativo, sexo, bebedor vs. no bebedor, fumador vs. no fumador y deportistas vs. no deportista)

\begin{tabular}{|c|c|c|c|c|c|c|c|c|c|c|c|c|}
\hline \multirow[b]{2}{*}{ Primer ciclo ESO } & \multicolumn{2}{|c|}{$\begin{array}{c}\text { BEM } \\
\text { Media (DT) }\end{array}$} & \multicolumn{2}{|c|}{$\begin{array}{c}\text { BEX } \\
\text { Media (DT) }\end{array}$} & \multicolumn{2}{|c|}{$\begin{array}{c}\text { DES } \\
\text { Media (DT) }\end{array}$} & \multicolumn{2}{|c|}{$\begin{array}{c}\text { SAB } \\
\text { Media (DT) }\end{array}$} & \multicolumn{2}{|c|}{$\begin{array}{c}\text { EBS-J } \\
\text { Media (DT) }\end{array}$} & \multicolumn{2}{|c|}{$\begin{array}{c}S \\
\text { Media (DT) }\end{array}$} \\
\hline & 4.70 & $(3.04)$ & 3.39 & (1.72) & 2.38 & (1.99) & 4.00 & $(2.47)$ & 14.46 & (5.97) & 6.18 & (2.46) \\
\hline Segundo ciclo ESO & 5.66 & (3.09) & 4.45 & $(1.82)$ & 4.35 & $(2.32)$ & 4.10 & $(2.34)$ & 18.56 & $(5.82)$ & 7.12 & $(2.26)$ \\
\hline p-valor & \multicolumn{2}{|c|}{0.005} & \multicolumn{2}{|c|}{$<0.001$} & \multicolumn{2}{|c|}{$<0.001$} & \multicolumn{2}{|c|}{0.824} & \multicolumn{2}{|c|}{$<0.001$} & \multicolumn{2}{|c|}{0.001} \\
\hline Niño & 6.26 & $(2.78)$ & 3.99 & (1.77) & 3.95 & $(2.35)$ & 3.77 & $(2.22)$ & 17.96 & (5.79) & 7.12 & (2.16) \\
\hline Niña & 4.48 & (3.14) & 4.18 & (1.93) & 3.43 & $(2.42)$ & 4.34 & $(2.50)$ & 16.42 & $(6.44)$ & 6.51 & (2.52) \\
\hline p-valor & \multicolumn{2}{|c|}{$<0.001$} & \multicolumn{2}{|c|}{0.556} & \multicolumn{2}{|c|}{0.052} & \multicolumn{2}{|c|}{0.050} & \multicolumn{2}{|c|}{0.027} & \multicolumn{2}{|c|}{0.032} \\
\hline Bebedor de alcohol & 5.54 & (3.08) & 4.31 & $(1.82)$ & 4.23 & (2.33) & 4.19 & $(2.34)$ & 18.25 & (5.95) & 7.18 & $(2.15)$ \\
\hline No bebedor & 4.70 & (3.09) & 3.42 & $(1.80)$ & 1.99 & $(1.75)$ & 3.70 & $(2.48)$ & 13.80 & $(5.67)$ & 5.64 & $(2.63)$ \\
\hline p-valor & \multicolumn{2}{|c|}{0.056} & \multicolumn{2}{|c|}{0.001} & \multicolumn{2}{|c|}{$<0.001$} & \multicolumn{2}{|c|}{0.150} & \multicolumn{2}{|c|}{$<0.001$} & \multicolumn{2}{|c|}{$<0.001$} \\
\hline Fumador & 5.72 & $(2.94)$ & 4.81 & $(1.83)$ & 4.92 & $(2.43)$ & 4.47 & $(2.34)$ & 19.91 & (5.71) & 7.49 & (2.15) \\
\hline No fumador & 5.08 & $(3.18)$ & 3.62 & $(1.72)$ & 2.87 & $(2.01)$ & 3.81 & $(2.37)$ & 15.37 & $(5.82)$ & 6.35 & $(2.40)$ \\
\hline p-valor & \multicolumn{2}{|c|}{0.096} & \multicolumn{2}{|c|}{$<0.001$} & \multicolumn{2}{|c|}{$<0.001$} & \multicolumn{2}{|c|}{0.025} & \multicolumn{2}{|c|}{$<0.001$} & \multicolumn{2}{|c|}{$<0.001$} \\
\hline Deportista & 5.54 & (3.09) & 3.89 & (1.81) & 3.49 & $(2.31)$ & 3.93 & $(2.34)$ & 16.85 & $(6.21)$ & 6.71 & (2.42) \\
\hline No deportista & 4.71 & $(3.05)$ & 4.67 & (1.87) & 4.21 & (2.57) & 4.46 & $(2.47)$ & 18.05 & (6.04) & 7.06 & (2.22) \\
\hline p-valor & \multicolumn{2}{|c|}{0.053} & \multicolumn{2}{|c|}{0.002} & \multicolumn{2}{|c|}{0.030} & \multicolumn{2}{|c|}{0.112} & & 160 & & 89 \\
\hline
\end{tabular}

$\mathrm{DT}=$ Desviación típica. $\mathrm{BEM}=$ Búsqueda de emociones $. \mathrm{BEX}=$ Búsqueda de excitación. $\mathrm{DES}=$ Desinhibición. $\mathrm{SAB}=$ Susceptibilidad al aburrimiento. EBS-J $=$ Valoración global de la escala (Total EBS-J). S = Sinceridad. ESO = Educación secundaria obligatoria. 
En el cuadro 2 se muestran los resultados de la escala EBS-J en relación con los diferentes factores explicativos. Los adolescentes de segundo de la ESO presentan puntuaciones significativamente mayores $(\mathrm{p}<0.01)$ en las dimensiones BEM, BEX, DES, total escala EBS-J y S. Los niños presentan puntuaciones significativamente mayores en $B E M(p<0.001)$, $\mathrm{S}(\mathrm{p}=0.032)$ y en el total de la escala EBS-J $(\mathrm{p}=0.027)$ que las niñas y cercano a la significatividad estadística en DES $(p=0.052)$ y las niñas mayor puntuación en $\mathrm{SAB}(\mathrm{p}=0.050)$. Los consumidores de alcohol presentan mayor puntuación en BEX ( $p=0.001)$, DES $(p<0.001)$, en la escala total EBS-J $(p<0.001)$ y en $S(p<0.001)$ y cercano a la significatividad estadística en BEM ( $p=0.056)$. Los fumadores muestran mayor puntuación que los no fumadores en BEX $(p<0.001)$, DES $(p<0.001)$, SAB $(P=0.025)$, en la escala total EBS-J $(p<0.001)$ y en $S(p<0.001)$. Por último, los no deportistas puntúan más que los deportistas en BEX ( $p=0.002)$ y DES $(p=0.030)$ y los deportistas mayores valores cercanos a la significatividad estadística en BEM ( $\mathrm{p}=0.053)$.

Del análisis de cluster, exponemos tres conglomerados diferenciados de mayor a menor puntuación en la escala EBSJ. En el conglomerado 1 se asignan 70 sujetos (25.74\%) siendo el grupo de mayor puntuación en la escala EBS-J (24.8); el conglomerado 2, con una puntuación intermedia (17.7) y 120 sujetos $(44.11 \%$ ) y el conglomerado 3 , con 82 sujetos $(30.15 \%)$ y con la puntuación más baja en la escala EBS-J (9.76).

En el cuadro 3 se exponen los resultados en el consumo de alcohol, consumo de tabaco y práctica deportiva en los diferentes conglomerados. Existe mayor presencia de fumadores y bebedores de alcohol conforme se asciende en el conglomerado con mayor valor en la escala EBS-J; sin embargo, encontramos un semejante porcentaje de deportistas en los tres conglomerados.

La edad se correlaciona de manera significativa con BEM $(r=0.152, p=0.012), \quad B E X \quad(r=0.315, p<0.001)$, DES $(\mathrm{r}=0.367, \mathrm{p}<0.001), \mathrm{S}(\mathrm{r}=0.127, \mathrm{p}=0.037)$ y escala total EBS-J $(\mathrm{r}=0.327, \mathrm{p}<0.001)$. El número de cigarros consumidos por semana se correlaciona significativamente con BEX $(r=0.224$, $\mathrm{p}<0.001)$, DES $(\mathrm{r}=0.319, \mathrm{p}<0.001), \mathrm{S}(\mathrm{r}=0.122, \mathrm{p}=0.044)$ y esca-

Cuadro 3. Consumo de alcohol, consumo de tabaco y práctica deportiva en los diferentes conglomerados

\begin{tabular}{lccccccc}
\hline & Alta BS & \multicolumn{2}{c}{ Media BS } & \multicolumn{2}{c}{ Baja BS } \\
& $\mathrm{n}(\%)$ & $\mathrm{n}(\%)$ & $\mathrm{n}(\%)$ & p-valor \\
\hline Bebedor & 63 & $(90.0)$ & 93 & $(77.5)$ & 49 & $(59.8)$ & $<0.001$ \\
No bebedor & 7 & $(10.0)$ & 27 & $(22.5)$ & 33 & $(40.2)$ & \\
Fumador & 42 & $(60.0)$ & 46 & $(38.3)$ & 19 & $(23.2)$ & $<0.001$ \\
No fumador & 28 & $(40.0)$ & 74 & $(61.7)$ & 63 & $(76.8)$ & \\
Deportista & 50 & $(71.4)$ & 89 & $(74.2)$ & 63 & $(76.8)$ & 0.738 \\
No deportista & 20 & $(28.6)$ & 31 & $(25.8)$ & 19 & $(23.2)$ & \\
\hline
\end{tabular}

BS. Búsqueda de sensaciones.

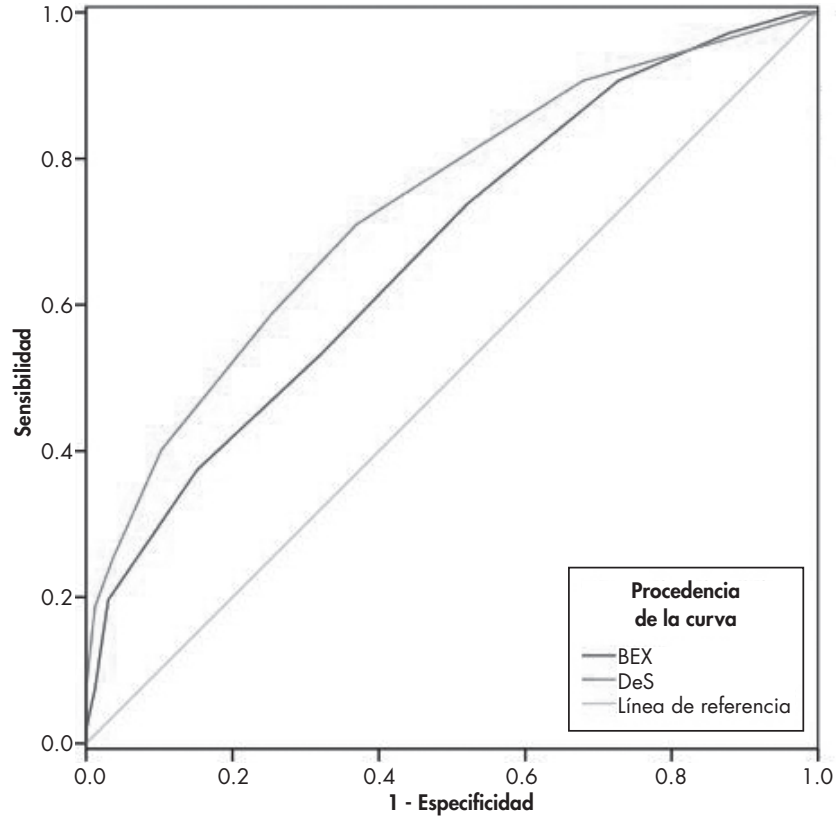

Figura 1. Curva ROC del hábito de fumar predicho por la DES (desinhibición) y la BEX (búsqueda de excitación).

la total EBS-J $(\mathrm{r}=0.199, \mathrm{p}=0.001)$. La frecuencia de bebida se correlaciona significativamente con BEX $(r=0.264, p<0.001)$, DES $(\mathrm{r}=0.495, \mathrm{p}<0.001), \mathrm{S}(\mathrm{r}=0.312, \mathrm{p}<0.001)$ y escala total EBS-J $(r=0.337, \mathrm{p}<0.001)$.

El análisis de regresión logística muestra que, en el caso del hábito de no ser deportista, el sexo femenino (odds ratio $=10.466$, I.C. $95 \%=4.919-22.268, \mathrm{p}<0.001)$ y la DES (odds ratio $=1.221$, I.C. $95 \%=1.074-1.387, \mathrm{p}=0.002$ ) son factores de riesgo. Para el hábito de fumar, la BEX (odds ratio $=1.204$, I.C. $95 \%=1.019-1.422, \mathrm{p}=0.030$ ), la DES (odds ratio $=1.318$, I.C. $95 \%=1.148-1.513, p<0.001)$ y el grupo de segundo ciclo de la $\mathrm{ESO}$ (odds ratio $=3.552$, I.C. $95 \%=1.781-7.083, \mathrm{p}<0.001$ ) son factores de riesgo. Por último, en el hábito de consumo de alcohol, la DES (odds ratio $=1.466$, I.C. $95 \%=1.227-1.752, p<0.001$ ) y el grupo de segundo ciclo de la ESO (odds ratio=7.854, I.C. $95 \%=3.935-15.676, \mathrm{p}<0.001)$ son factores de riesgo.

El hábito de no ser deportista predicho por la DES presenta un bajo poder de discriminación ( $\mathrm{ABC}$ [área bajo la curva $=0.573$, I.C. $95 \%=0.495-0.651, p=0.070$ ). La figura 1 muestra la curva ROC del hábito de fumar predicho por la DES $(\mathrm{ABC}=0.735$, I.C. $95 \%=0.674-0.796, \mathrm{p}<0.001)$ y la BEX $(A B C=0.673$, I.C. $95 \%=0.608-0.738, p<0.001)$, situándose el punto de corte para la DES en 4.50 (sensibili$\mathrm{dad}=0.589,1$-especificidad $=0.255$ ) y para la BEX en 4.50 (sensibilidad $=0.533,1$-especificidad $=0.321$ ), con lo que resulta un aceptable poder de discriminación para ambas variables. Por último, y en relación con el hábito de consumo de alcohol, en la figura 2 se muestra la curva ROC en la que la DES $(\mathrm{ABC}=0.776$, I.C. $95 \%=0.715-0.837, \mathrm{p}<0.001$ ) proporciona un alto poder de discriminación, situándose el punto de corte en 2.50 (sensibilidad $=0.761$, 1 -especificidad $=0.328$ ). 


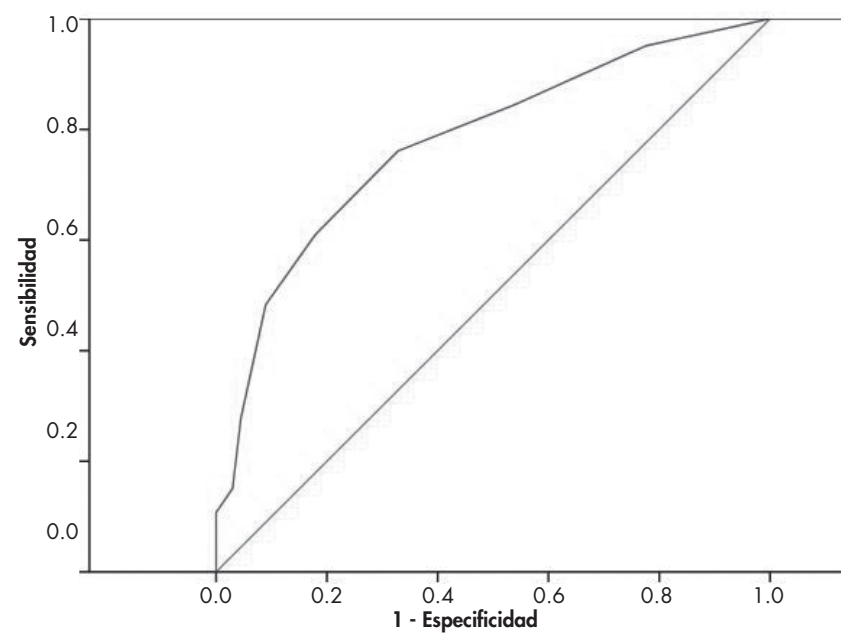

Figura 2. Curva ROC del hábito de consumo de alcohol predicho por la DES (desinhibición).

\section{DISCUSIÓN}

La BS en sus dimensiones BEX y DES es un factor moderadamente predictivo del consumo de tabaco y DES igualmente en el consumo de alcohol. Ninguna dimensión de la escala EBS-J mostró capacidad predictiva de la conducta sedentaria en adolescentes. En este estudio, el 76\% de los niños y el 75.4\% de las niñas se declaran bebedores de alcohol $(p=0.906)$. En el consumo de tabaco, el 36.2\% de los niños y el $42.3 \%$ de las niñas fuman $(\mathrm{p}=0.304)$. Datos similares al estudio de Cándido et al. (2007), ${ }^{9}$ que indican que el $82.20 \%$ de los niños han consumido alguna vez alcohol y el $40.40 \%$ tabaco. Estos resultados corroboran los hallazgos del Observatorio Español sobre Drogas ${ }^{8}$ que indican que un $81.2 \%$ de los niños había tomado bebidas alcohólicas alguna vez en la vida y un $44.6 \%$ tabaco. También son semejantes con los datos del European School Survey Project on Alcohol and Drugs ${ }^{29}$ que indican que a los 15 años el $30 \%$ de los escolares europeos fumaron al menos un cigarrillo.

En consonancia con otros estudios,,$^{30}$ esta investigación muestra la existencia de un gran número de adolescentes consumidores de alcohol y tabaco, lo que evidencia una gran prevalencia de consumo de estas sustancias adictivas. La edad es un factor de riesgo de tabaquismo y consumo de alcohol. De este modo, los adolescentes del segundo ciclo de la ESO presentan mayor consumo; por el contrario, el sexo no fue un factor predictivo del consumo. Sin embargo, en España, ser mujer es un factor de riesgo en el consumo frecuente de bebidas alcohólicas. ${ }^{30}$ Respecto del consumo de tabaco, se aprecia una mayor probabilidad de consumo entre las alumnas. A medida que aumenta la edad, se acrecienta la probabilidad de consumir alcohol y tabaco con más frecuencia. ${ }^{30}$

La propensión al uso de sustancias como el alcohol, el tabaco y la marihuana está asociada a la BS. ${ }^{31}$ En este estudio, los adolescentes que fuman y beben alcohol presentan una puntuación más alta en la escala total EBS-J. En este sentido, la BS es mayor en los hombres y mujeres que presentan consumo de nicotina y alcohol. ${ }^{31}$ Saiz et al. (1999) ${ }^{32}$ también destacan una mayor puntuación en la escala de BS entre jóvenes (tanto hombres como mujeres) consumidores de sustancias ilegales. Por su parte, Sargent et al. $(2010)^{33}$ muestran que la BS es una variable predictiva tanto del consumo excesivo de alcohol en adolescentes hombres (ABC $=0.72$, IC 95\% $=0.69-0.74)$ como en mujeres $(\mathrm{ABC}=0.70$, IC 95\% $=0.68-0.73)$ y del consumo de tabaco en hombres (ABC $=0.78$, IC 95\% $=0.74-0.83$ ) como en mujeres $(\mathrm{ABC}=0.81$, IC 95\%=0.75-0.86). Además, en este estudio, en consonancia con otros autores, ${ }^{34,35}$ la BS se asoció positivamente con el desarrollo puberal en hombres y mujeres.

El 92.3\% de los niños por el 57.7\% de las niñas $(p<0.001)$ sí son deportistas, práctica que se reduce con la edad. Así, el $83.9 \%$ de los niños del primer ciclo de la ESO por el $69.3 \%$ en el segundo ciclo se declaran deportistas $(p=0.009)$. Estos resultados son superiores a los de Román et al. (2006), ${ }^{36}$ que indican que el 39\% de los chicos y el 21\% de las chicas de entre dos a 24 años practican actividad física dos o más días a la semana. Estos datos son superiores a los encontrados en la Encuesta Nacional de Salud (2006), ${ }^{37}$ en la cual, para la franja de edad de 16 a 24 años, existe una prevalencia de actividad física del $71.8 \%$ en hombres y del $55.5 \%$ en mujeres. En este estudio, ser mujer es un factor de riesgo de sedentarismo. Otros autores ${ }^{38}$ reflejan igualmente que el sexo (masculino) y la edad (a mayor edad menor práctica deportiva) son variables que se asocian de forma consistente con la actividad física de los adolescentes, además de otras variables como la competencia percibida, la actividad física anterior, los deportes de la comunidad, la BS, el apoyo de los padres, el apoyo de los demás, la actividad física entre hermanos y las oportunidades para hacer ejercicio. Con la edad se produce un aumento de la actividad física hasta los 10-13 años; a partir de entonces disminuye su práctica. El nivel socioeconómico y el nivel de estudios de la madre influyen positivamente en el grado de actividad física de la población. ${ }^{36}$ La relación entre el consumo de alcohol o tabaco y los comportamientos ante la práctica físico-deportiva señalan que los adolescentes físicamente activos fuman y beben menos que los inactivos, resultados en consonancia con Ruiz-Risueño et al. (2012). ${ }^{30}$

La relación entre la BS, el desarrollo puberal y los hábitos de vida, en particular el consumo de alcohol y de tabaco en adolescentes, puede contribuir a comprender los cambios en el uso de drogas que se observan en esta edad. La eficacia del uso de la excitación alternativa, por ejemplo, mediante la práctica de determinados deportes todavía no se ha determinado y sería una fructífera línea de investigación. Es necesario tener en cuenta que la validez predictiva de los factores de riesgo en el consumo de alcohol, tabaco y conducta sedentaria debe analizarse en muestras longitudinales antes de que se emplee como variable de orientación, con el 
fin de evaluar la validez predictiva general y determinar el correspondiente valor de corte. Estos resultados ponen de relieve la importancia de atender y evaluar adecuadamente los cambios en rasgos relacionados con la adolescencia temprana como predictores singulares de inicio y la progresión del consumo de sustancias y hábitos de vida. Los resultados del estudio también tienen potenciales implicaciones clínicas, específicamente al establecer el fundamento adicional para el desarrollo de intervenciones para la BS y la propensión al riesgo como un medio para reducir el consumo de sustancias.

\section{Limitaciones}

Este estudio es un primer paso en la comprensión del cambio en la BS en relación con el consumo de alcohol, tabaco y hábito de práctica deportiva en la adolescencia temprana, aunque se han examinado un número limitado de covariables. La investigación futura se beneficiaría del análisis de otras características teóricamente importantes (por ejemplo: expectativas con respecto al uso de sustancias, la vigilancia de los padres, estilos parentales, los acontecimientos vitales negativos, la influencia del entorno social y los amigos).

\section{CONCLUSIONES}

La BS es un factor de personalidad relacionado con el consumo temprano de sustancias como el alcohol y el tabaco, y algunos de sus rasgos se revelan como factores predictivos del consumo de estas sustancias. Sin embargo, la BS no se ha asociado al hábito de práctica deportiva en adolescentes. Los deportistas consumen menos alcohol y tabaco.

\section{REFERENCIAS}

1. Rodrigo MA, Máiquez ML, García $M$, Mendoza $R$ et al. Relaciones padres-hijos y estilos de vida en la adolescencia. Psicothema 2004;16(2):203-210.

2. Mantilla SC, Gómez A, Hidalgo MD. Physical activity and tobacco and alcohol use in a group of university students. Rev Salud Pública (Bogotá) 2011;17(5):748-758.

3. Moreno JA, Moreno R, Cervelló, E. Relación de autoconcepto físico con las conductas de consumo de alcohol y tabaco en adolescentes. Adicciones 2009;21(1):147-154.

4. Ruiz-Risueno J, Ruiz-Juan F, Zamarripa JI. Alcohol and tobacco consumption in Spanish and Mexican adolescents and its relation to physical and sports-related activity and to the family. Rev Panam Salu d Publ 2012;31(3):211-220.

5. Terry-McElrath YM, O'Malley PM, Johnston LD. Exercise and substance use among American youth, 1991-2009. Am J Prev Med 2011; 40(5):530-540.

6. Aranceta J, Serra L, Foz M, Moreno B, Grupo Colaborativo SEEDO. Prevalence of obesity in Spain. Medi Clin-Barcelona 2005;125(12):460-466.

7. Tercedor P, Martín-Matillas P, Chillón LJ, Pérez FB et al. Incremento del consumo de tabaco y disminución del nivel de práctica de actividad física en adolescentes españoles. Nutr Hosp 2007;22(1):89-94.
8. Ministerio de Sanidad y Política Social. Evaluación final de la estrategia nacional sobre drogas 2000-2008. Madrid: Delegación del Gobierno para el Plan Nacional sobre Drogas; 2010.

9. Cándido J, Delgado B, Bautista R, Torregrosa M et al. Factores psicosociales relacionados con el consumo de alcohol y tabaco en adolescentes españoles. Int J Clin Hlth Psyc 2007;7(2):403-420.

10. Ruiz F, Ruiz J. Variables predictoras de consumo de alcohol entre adolescentes españoles. An Psicol-Spain 2011;27(2):350-359.

11. Lorente FO, Souville M, Griffet J, Grélot L. Participation in sports and alcohol consumption among french adolescents. Addict Behav 2004;29(5):941-946.

12. Pastor $Y$, Balaguer I, García-Merita M. Relaciones entre el autoconcepto $\mathrm{y}$ el estilo de vida saludable en la adolescencia media: un modelo exploratorio. Psicothema 2006;18(1):18-24.

13. Bovard RS. Risk behaviors in high school and college sport. Curr Sport Med Rep 2008;7(6):359-366.

14. Dunlop SM, Romer D. Adolescent and young adult crash risk: sensation seeking, substance use propensity and substance use behaviors. J Adolescent Health 2010;46(1):90-92.

15. Roberti JW, Storch EA, Bravata E. Sensation seeking, exposure to psychosocial stressors, and body modifications. Pers Indiv Differ 2004;37(6):1167-1177.

16. Zuckerman M. Sensation seeking and risky behaviour. Washington, DC: American Psychological Association; 2007.

17. Zuckerman M. Sensation seeking: Beyond the optimal level of arousal. Hillsdale, NJ: Erlbaum; 1979.

18. Zuckerman M, Eysenck S, Eysenck HJ. Sensation seeking in England and America: Crosscultural, age, and sex comparisons. J Consult Clin Psych 1978;46(1):139-149.

19. Chico L. Búsqueda de sensaciones. Psicothema 2000;12(2):229-235.

20. Stoel RD, De Geus EJ, Boomsma DI. Genetic analysis of sensation seeking with an extended twin design. Behav Genet 2006;36(2):229-237.

21. Gullo MJ, Dawe S. Impulsivity and adolescent substance use: Rashly dismissed as "all-bad"? Neurosci Biobehav R 2008;32(8):1507-1518.

22. Urban R. Smoking outcome expectancies mediate the association between sensation seeking, peer smoking, and smoking among young adolescents. Nicotine Tob Res 2010;12(1):59-68.

23. Crawford A, Pentz M, Chou C, Li C et al. Parallel developmental trajectories of sensation seeking and regular substance use in adolescents. Psychol Addict Behav 2003;17(3):179-192.

24. Guszkowska M, Boldak A. Sensation seeking in males involved in recreational high risk sports. Biol Sport 2010;27(3):157-162.

25. Oliva A. Desarrollo cerebral y asunción de riesgos durante la adolescencia. Apuntes Psicología 2007;25(3):239-254.

26. Pérez J, Ortet G, Plá S, Simó S. Escala de búsqueda de sensaciones para niños y adolescentes (EBS-J). Evaluación Psicológica 1987;3(2):283-290.

27. Currie C. Health behaviour in school-aged children. Research protocol for the 1997-98 survey. A World Health Organization Cross-National Study. Edinburgh: University of Edinburgh; 1998.

28. Haskell WL, Lee IM, Pate RR, Powell KE et al. Physical activity and public health: updated recommendation for adults from the American College of Sports Medicine and the American Heart Association. Med Sci Sports Exerc 2007;39(8):1423-1434.

29. Hibell B, Anderson B, Ahlstrom S, Balakireva O et al. Alcohol an other drug use among students in 30 european countries. Alcohol Alcoholism 2003;38(1):99.

30. Ruiz J, Ruiz F, Zamarripa JI. Alcohol and tobacco consumption in Spanish and Mexican adolescents and its relation to physical and sports-related activity and to the family. Rev Panam Salu Publ 2012;31(3):211-220.

31. Martin CA, Kelly TH, Rayens MK, Brogli BR et al. Sensation seeking, puberty, and nicotine, alcohol, and marijuana use in adolescence. $J$ Am Acad Child Adolesc Psychiatry 2002;41(12):1495-1502.

32. Saiz PA, González MP, Jiménez L, Delgado Y et al. Consumo de alcohol, tabaco y otras drogas y rasgos de personalidad en jóvenes de enseñanza secundaria. Adicciones 1999;11(3):209-220. 
33. Sargent JD, Tanski S, Stoolmiller M, Hanewinkel R. Using sensation seeking to target adolescents for substance use interventions. Addiction 2010;105(3):506-514.

34. Kelly AB, Chan G, Toumbourou JW, O'Flaherty M et al. Very young adolescents and alcohol: Evidence of a unique susceptibility to peer alcohol use. Addictive Behaviors doi:10.1016/j.addbeh.2011.11.038.

35. MacPherson L, Magidson JF, Reynolds EK, Kahler CW et al. Changes in sensation seeking and risk-taking propensity predict increases in alcohol use among early adolescents. Alcohol Clin Exp Res 2010;34(8):1400-1408.
36. Román B, Serra L, Ribas L, Pérez-Rodrigo C et al. Actividad física en la población infantil y juvenil española en el tiempo libre. Apunts Med L'esport. 2006;41(151):86-94.

37. Ministerio de Sanidad y Consumo. Encuesta Nacional de Salud 2006. Recuperado de http:/www.msps.es/estadEstudios/estadisticas/encuestaNacional/encuestaNac2006/encuestaNacionalSalud2006.pdf.

38. Sallis JF, Prochaska JJ, Taylor WC. A review of correlates of physical activity of children and adolescents. Med Sci Sport Exer 2000;32(5):963-975.

Artículo sin conflicto de intereses 